\title{
Influences of combining nano zinc, honey and Aloe vera to accelerate healing the wounds caused by third-degree burn in male balb/c mice
}

\author{
Reza Yari $^{1 *}$, Iraj Khodadadi ${ }^{2}$, Farshid Aliyari ${ }^{3}$, Zahra Saremi ${ }^{4}$
}

1. Department of Biology, Faculty of Sciences, Borujerd Branch, Islamic Azad University, Borujerd, Iran

2. Department of Biochemistry, School of Medicine, Hamedan University of Medical Sciences, Hamedan, Iran

3. School of Medicine, Lorestan University of Medical Sciences, Khorramabad, Iran

4. Young Researchers and Elite Club, Borujerd Branch, Islamic Azad University, Borujerd, Iran

*Corresponding author:Tel: +98 9127461696 Fax: +98 -

Address: Department of Biology, Faculty of Sciences, Borujerd Branch, Islamic Azad University,

Borujerd, Iran

E-mail: rezayari@yahoo.com,rezayari@iaub.ac.ir

Received; 2017/05/5 revised; 2017/06/10 accepted; 2017/07/13

\begin{abstract}
Introduction: Burns are one of the most common household and industrial injuries. There are evidences which demonstrate the therapeutic properties of honey and Aloe vera. We evaluated the topical influences of this material and nano zinc combination on healing the wounds caused by third-degree burns.

Materials and methods: $32 \mathrm{balb} / \mathrm{c}$ mice divided into a control group (without treatment), group 1 (treated with Aloe vera and nano zinc), group 2 (treated with Aloe vera, honey and nanoz inc) and group 3 (treated with honey and nano zinc). The third-degree burn was created on the back of balb/c mice with general anesthesia observing sterile conditions. Local treatment of burn was conducted once a week during 6 weeks and after the end of treatment, were anesthetized by ether and then killed. After fixation, the practical steps of general histology technique were performed on it. The samples stained with hematoxylin-eosin and they observed with a microscope.

Results: We found full tightening of the burn wound and less scar in the group treated with nano zinc and honey compared to control group and other groups. In histological studies, a significant increase was found in the overall thickness of the skin, keratinocyte layer, the epidermis and hypodermis, number and diameter of the hair follicles in a third group versus other groups.

Conclusion: The results showed the organic honey and nano zinc combination accelerate the healing process of burn wound in male balb/c mice. While adding Aloe vera to this composition doesn't have an effect on wound healing.
\end{abstract}

Keywords: Nano zinc, Honey, Aloe vera, balb/c mouse, Burns

\section{Introduction}

Aloe vera is a medicinal plant which has been used since $1500 \mathrm{BC}$ in many countries such as Greece, China, and Mexico and used for centuries in these

Copyright (C) 2018 Journal of Basic Research in Medical Science. This is an open access article distributed under the terms of the Creative Commons Attribution 4.0 International License (https://creativecommons.org/licenses/by/4.0/) which permits copy and redistribute the material, in any medium or format, provided the original work is properly cited. 
centuries as a topical drug for various diseases and skin lesions treatment (1). Up to now, 75 constituents were found in Aloe vera including 20 minerals, 20 kinds of amino acid, 12 kinds of vitamins and water (2). This phytogel contains various constituents such as polysaccharide glucomannan, carboxypeptidase, magnesium, zinc, calcium, glucose, cholesterol, salicylic acid, prostaglandins, vitamin $\mathrm{A}, \mathrm{C}$ and $\mathrm{E}$ and lignin, plant sterols and amino acids and some enzymes including alloyed, alkaline phosphatase, amylase, brady kinase, carboxypeptidase, catalase, cellulase, lipase and peroxidase. Its regenerative feature mostly relates to a compound called glucomannan that is rich in polysaccharides like mannose. glucomannan effects on receptors of fibroblast growth factor and stimulate its activity and proliferation which in turn increases the production of Collagen. Aloe vera gel not only increases the wound collagen rate but also changes the composition of collagen as well as collagen crosslinking. As a result, it accelerates wound healing. Similarly, it has been found that Aloe vera accelerates the immune system activity against vast ranges of bacteria including Pseudomonas aeruginosa, Escherichia coli, Salmonella typhimurium, Klebsiella pneumoniae, Mycobacterium tuberculosis, Streptococcus pyogenes, Serratia, Staphylococcus aureus. Using Honey to treat wound was very common in medicine of countries such as Greece, Egypt and India and recently it is introduced as a treatment factor $(3,4-7)$. Honey is a saturated mixture consisting of monosaccharides, Fructose and glucose that leaves a little water for growing pathogenic microorganisms and on the other hand it has antibacterial property due to $\mathrm{pH} 2.3$ to 5.4. Antimicrobial properties of Honey result from the ability to produce Hydrogen peroxide (8). The concentration of Hydrogen peroxide in Honey is 1 $\mathrm{mmol} / \mathrm{L}$ that is activated and released with dilution slowly which does not hurt tissues
(9). In the other hand, the growth of cells responsible for replacement simulates damaged tissue which has insulin-like effect on the cells involved in wound healing that stimulates the proliferation of new blood vessels and activates protein digestive enzymes in involved tissues. Honey's ability to suppress inflammation may be related to its ability to suppress free radicals (10). Anti-oxidant inhibits the free radicals generation responsible for anti- inflammatory effect of honey and provides a moist environment Anti-oxidant which inhibits the free radicals generation which is responsible for anti-inflammatory effect of Honey and provides a without risk moist environment for wound healing and growth of bacteria. Its antiinflammatory properties reduce edema, exudate and wound malodor (8). Honey contains a mixture of sugar and other substances. Its main carbohydrates include fructose $(38 \%)$ and glucose $(31 \%)$. The rest of its carbohydrates include maltose, sucrose, and other carbohydrates. Honey contains multiple vitamins including B6 and $\mathrm{C}$, thiamine, niacin, riboflavin, pantothenic acid and the minerals such as calcium, iron, magnesium, manganese, phosphorus, potassium, sodium, zinc and amino acids. Antioxidant compounds in honey contain chrysin, pino baksin, pinocembrin, vitamin $\mathrm{C}$ and catalase (8). Vitamin $\mathrm{C}$ content of honey is three times more than a plasma that feeds the regenerating tissue. Honey acts as a mitogenic factor toward $B$ and $T$ lymphocytes $(8,10)$. Honey does not have identical antimicrobial and chemical properties. Antimicrobial property of honey can effect on wound healing individually since it inhibits the growth of bacteria and generation of metabolism products and damages tissue. Honey, accelerate wound healing, angiogenesis, granulation and epithelialization $(8,11)$. Wound recovery takes place with a minimal scar that does not have a harmful effect and rarely is allergic and irritating. The insertion and removal of dressing are 
very easy (11).Nanoparticles due to their unique optical, electrical and mechanical characteristics unlike bulk form matter attracted the attention of many researchers. These nanoparticles have numerous usages in catalytic activity, fluorescent, anti-odor removing bacteria, cosmetics, gas sensors, ultraviolet light absorbing materials, piezoelectric devices, photodiodes, solar cells, light-emitting diodes, lasers, paints, varnishes, and rubber manufacturing industry. Zinc ultra-fine particles are very transparent, and so they are used in the production of sunscreens, paints, varnishes, plastics and cosmetics especially to prevent the passage of the glasses wave. Recently zinc oxide nanoparticles were used in the production of sunscreen glass. When the particle size is in nanometer limit, one of the downsizing influences is impressionability against light and electromagnetic. Chemical properties, physical microstructural and zinc oxide powder relates to their synthesis method. Thus due to extensive application of zinc oxide in advanced technologies in the past years, researchers have mostly focused on synthesis method of zinc oxide nanoparticles to can synthesize these nanoparticles with intended properties (12). Zinc has an important role in the protein structure of zinc finger that also play an important role in regulating gene expression and cancer prevention. Use of fuzzy oxides nanoparticles in industry increases, human exposure to nanoparticles in the workplace and peripheral environment increase also. However, the effects of these nanoparticles on nervous system health thoroughly have investigated, so physiologic and pharmacologic assessment of this matter has special importance.

\section{Materials and methods}

In this study, 32 adult male albino balb/c mice in the weight range of 25 to $30 \mathrm{~g}$ were employed. The animals were examined as well to be sure that there is no infection in their bodies. These balb/c mice kept in an animal room with appropriate circumstances with the temperature of $2 \pm$ $22{ }^{\circ} \mathrm{C}$, relative humidity 40 to $60 \%$ and the 12-hour cycle of photoperiodic of the day. Their food included pellets for laboratory animals and tape drinking water. For years, different compounds are used to control the infection in burn wounds. In this study, oral Tetracycline antibiotics soluble in water were used in mice's drinking water. Due to the use of antibiotics as adjunctive therapy in the removal of specific microorganisms, overuse of tetracycline seems logical because of its positive effects on anaerobic gram-negative bacteria especially in very destructive items and cases which do not respond to conventional therapies. An interesting fact about Tetracycline is that synthetic and natural forms can inhibit antibacterial effects as well as reduce collagenase enzyme activity. In this study, above-mentioned antibiotic was added to drinking water of mice in powder form (1). In order to assimilate the treated animal condition, mice in the control group fed antibiotics similar to other groups. First, a mother solution was prepared from each matter individually to provide a laboratory material which its base is neutral lubricant gel. After preparing mother solutions according to selected materials used of each group, we combined a certain amount of solutions after accurate measurement and kept them in separate containers. We wrapped all containers in aluminum foil and put them in a fridge to stay away from the light. In this way, we extracted all material using a sterile syringe from mother solution and mixed them in a clean container, then put their cap and wrapped in a foil and kept in the fridge.

Mice randomly divided into four experimental groups that each one includes 8 mice's head. First mice were anesthetized by ether. In this way, we kept them in the closed and transparent chamber containing a high dose of ether. After anesthesia, we 
put them on canonization mattresses upon abdomen and shaved their hair by a razor. Shaved area disinfected with cotton dipped in ethanol. Then we put a 1000 Rial coin with a diameter of $2 \mathrm{~cm}$ heated for 3 minutes by a spirit lamp on mice back skin between the shoulder and the neck. We kept the coin 8 second on the abovementioned area of skin consequently similar strong burns were generated as thermal burns (Figure 1).

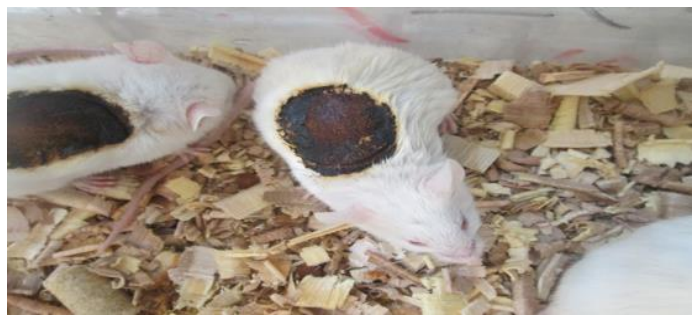

Figure 1. Third-degree burn in male mice.

Sterile physiological serum applied for burned place to measure disinfection of the treated material properties. The experimental groups included: (A) control group left without any treatment after strong burning, (B) group 1: including injured mice treated with Aloe vera and nano zinc, (C) group 2: including injured mice treated with Aloe vera, Honey and nano zinc, (D) group 3: including injured mice treated with Honey and nano zinc. Local treatments were implemented once a day at specific hours. At the end of each week, a mouse was selected from each group and after anesthetizing was dead. Considered area of the skin treated after burning was shaved with a shaver. After taking a photo of wound area in order to compare the amount and quality of macroscopic shrinkage and recovery of the wound from the macroscopic viewpoint, that area's skin was cut using a scalpel blade. After dehydration and preparing paraffin template, 6-micron sections (excision) took of samples and hematoxylin-eosin staining was performed on them. The aim of making incisions was measuring skin layer thickness and measuring the number and diameter of the hair follicles and hair shafts.

\section{Results}

Macroscopic findings: Macroscopic images are taken from wound place in experimental groups after passing treatment period confirm complete tightening of the wound in the third group treated with Honey and nano zinc versus the other group. Also, wound recovery and tightening process were better in the third group compared to two other group and control group. Topical treatment in a group treated with the combination of Honey and nano zinc (third group) left less scar compared to other groups.

Microscopic findings: Microscopic examination of tissue sections showed that the mixture of honey and nano zinc has a significant impact on third-degree burn recovery and amelioration is faster than control group and groups treated with other mixture and left less scar (Figure 3c). A remarkable point is that after observed microscopic assessments, it was found that all groups suffered from severe inflammation in the first three weeks but the third group did not observe any inflammation at the end of the third week. In this group, inflammation and edema removed at the end of the fourth week and any single-core or multi-core cells and red blood cells were not observed. The epidermis was generated and stratum corneum was also generating. In general, there is a significant difference between fifth group and three other groups in term of recovery rate and dermal fibroblasts count and epidermal situation (Figure 3). The overall thickness of the skin, keratinocyte layer, epidermis and hypodermis and a significant increase in the shaft, the number and diameter of the hair follicles in third group compared to the control group and two other controls, also a significant increase $(\mathrm{P}<0.05)$ in dermal thickness compared to the control group suggests the positive effects of Honey and nano zinc in accelerating the tightening in deep burn wounds and accelerating recovery and differentiation of 
skin layers and appendages.Dermis thickness in the group showed a significant increase in the control group $(\mathrm{P}<0.05)$ but

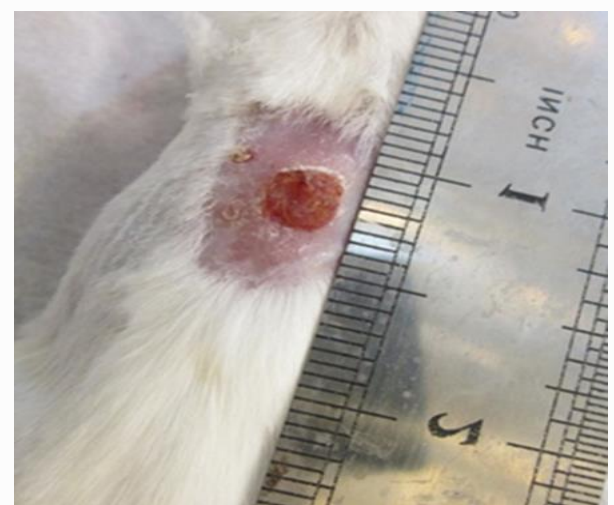

(a)

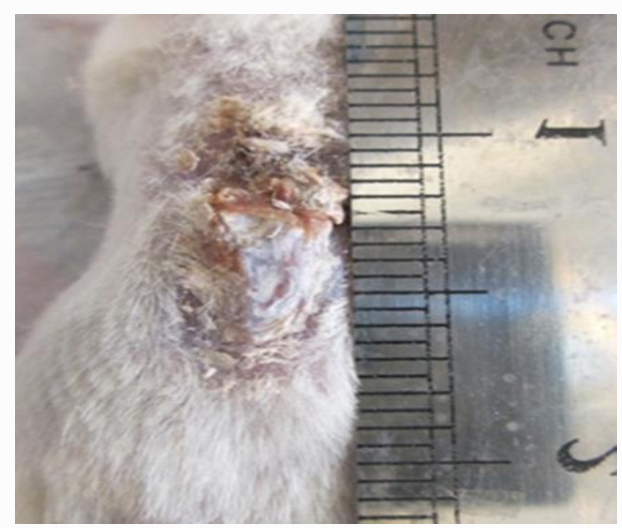

(c) was not observed any significant change in group 1 compared to the control group (Figure 3).

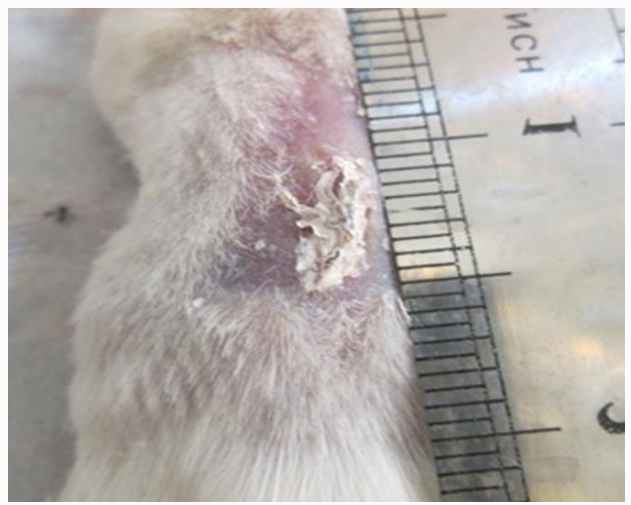

(b)

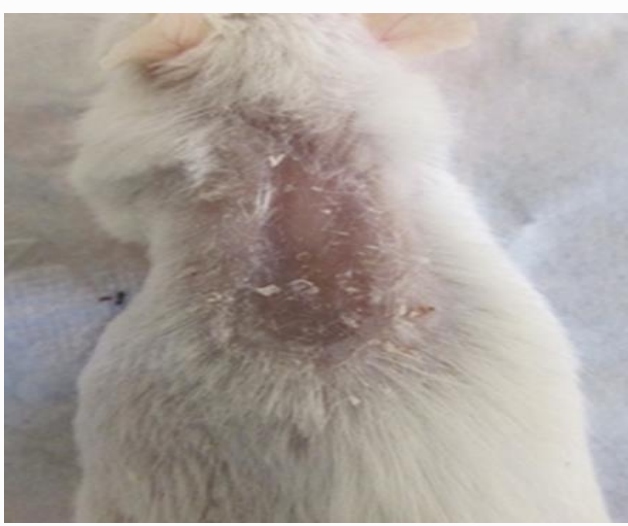

(d)

Figure 2. Wound shrinkage during 28 days without treatment: (a) The control group, (b) The group 1 (c) The group 2 and (d) The group 3.

Hypodermis thickness of the second and third group showed a significant increase compared to control group $(\mathrm{P}<0.05)$ (Figure 3, panels d and c).

The number of hair follicles in 3 group indicated a significant increase $(\mathrm{P}<0.05)$ compared to control group but did not have significant increase compared to 2 group. Hair follicle diameter in 3 group showed a significant increase compared to control group $(\mathrm{P}<0.05)$ but the observed increase of 2 group was not significant compared to control group (Figure 3).

\section{Discussion}

The tensile strength of sample tissue treated by Honey and nano Zinc (3 group $3^{\text {rd }}$ week) and 3 other groups show a significant difference relative to each other in term of wound healing rate and dermal fibroblasts count and epidermal. Studies show that the factors that increase blood flow and decrease inflammation and disinfection will have a positive effect on the recovery process of burn wound (15). It is demonstrated that tensile strength of skin mostly relates to Collagen fibers amount and how to connect this fiber to each other. The previous studies on hexose, fructose, sucrose, galactose and glucose in cell medium showed that these compositions are given that positive metabolic effects stimulate protein synthesis, collagen, extracellular matrix and growth factor. Astragalus honey probably due to having hexose and the different combination can increase collagen synthesis through increasing the 
fibroblasts, increasing the biosynthesis of nucleic acids and protein.

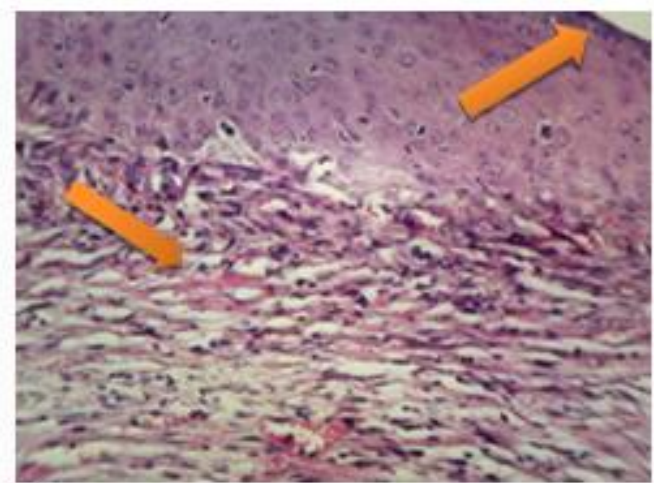

(a)

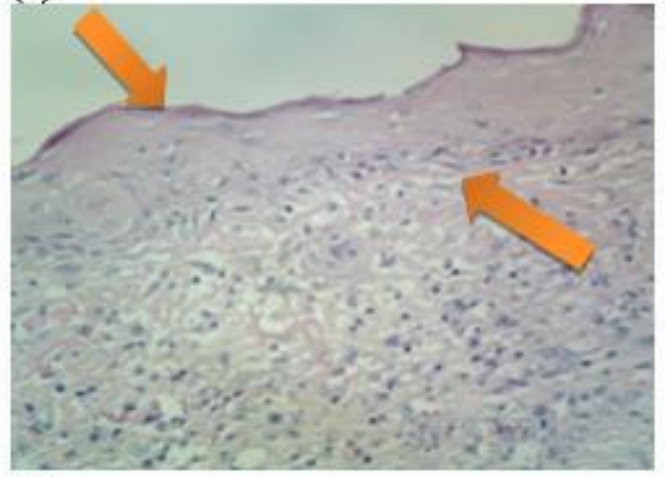

(c)

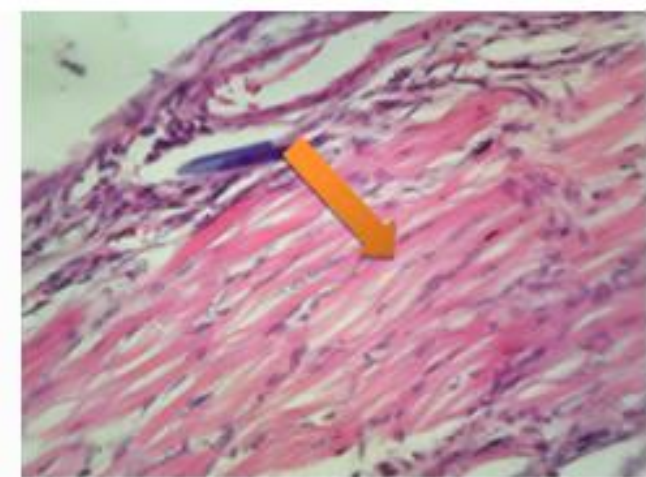

(b)

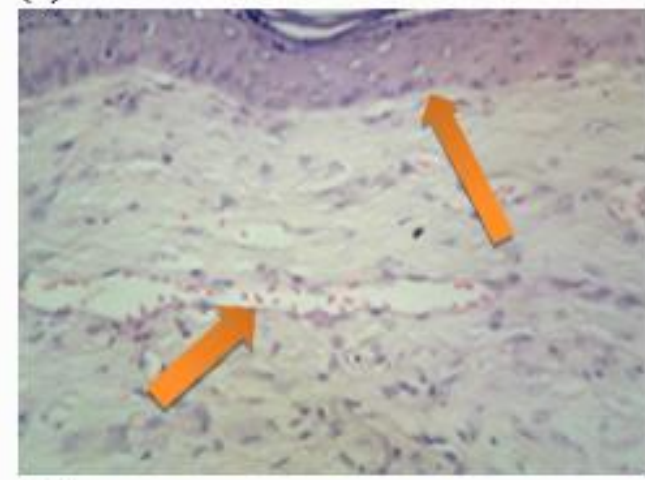

(d)

Figure 3. (H \& E stain) Photomicrographs of skin samples (magnification $40 \mathrm{X}$ ): (a) The group 1, formation of vascular tissue and epidermis, (b) The group 2, severe secretion of red blood cells as a result of inflammation, (c) The group 3, agglomeration of fibroblasts in the dermis, formation of a regular epidermis, dermis and cuticles, (d) The control group, formation of epidermis and the presence of red blood cells in the tissue.

In addition, natural honey can effect on puberty and orientation of collagen fibers positively and thus increase skin tensile. Huang and colleagues in 2002 showed that TGF- $\beta$ antagonists accelerate reducing scar result from burn wound and increasing wound recovery speed $(16,17)$. Copper and zinc ions are substances that their antimicrobial properties have been known traditionally from a long time ago. Some studies demonstrated that these metal ions react through sulfhydryl groups binding ( $\mathrm{SH}-$ ) in enzymes to proteins and finally proteins will be inactive. If these metals are present in very small size, they will show better antimicrobial properties due to increased surface proportional to volume ratio (18). Also, Atmaca and colleagues investigating the effect of zinc on the growth of microorganisms such as

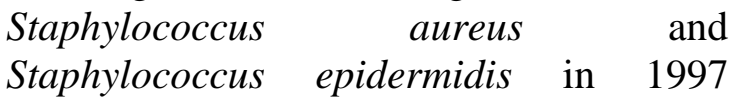

demonstrate that above mentioned items can suppress this microorganism. They will show better antimicrobial properties due to increased surface compared to volume ratio (19). Hsiao and colleagues investigated antimicrobial activity of copper particles against some bacteria. However, nanoparticles can be harmful to health due to two reasons. Firstly, it passes very fast through the biologic membrane and the second reason relates to its novelty, their toxicity still is not understood completely and their toxicity relates to the concentration and shape and diameter (20). More research is needed to investigate about nanoparticle and its effects on organ and blood factors. Also, applying laboratory mice as an animal model and different kinds of treatment and a nanoparticle with various compositions reveals new horizons for future research to examine the applications of 
nanotechnology in the physiology. According to the results of macroscopic and microscopic tests of this study and reports from another researcher can conclude that usage of natural honey due to biosynthesis increase of nucleic acids and proteins and synthesis of collagen and nano zinc with analgesic and antimicrobial properties can increase the healing wounds rate and increase the strength significantly. In fact, this project represents the design of a nanocomposite to improve healing burn wound in male mice. Angiogenic is an important factor in healing wound that during which wound area is saturated by blood vessels and this phenomenon is necessary to feed the wound and in the absence of it, recovery does not take place. The factor that stimulates angiogenesis can improve the normal healing process. An important part of wound healing results from angiogenesis and without it, macrophages and fibroblasts in the wound are not useful due to lack of oxygen and nutrients. The proliferation of blood vessels in the group treated with Honey and nano zinc (group 3) continues to $14^{\text {th }}$ day consistent with the proliferative phase and then it gradually decreases with the restricting phase consistently.

The increase in the third group and control group was significant $(\mathrm{P}<0.05)$. It seems that oxygen peroxide generated in Honey has therapeutic effects on treatment of wounds because cause growing cells which must be substitute wound tissue and has an insulin-like effect on wound healing. On the other hand, bacteria of wound area consume Honey glucose and produce lactic acid that plays a role in angiogenesis. Mandal believes that Honey accelerates angiogenesis and granular tissue formation in the wound area (22). The depth of granular tissue in $7^{\text {th }}$ day in group 3 is more than the control group and another group significantly $(\mathrm{P}<0.05)$ (The photoes were not shown). It seems that was to its nutritional properties accelerates wound recovery by the acceleration of granular tissue generation. The depth of most granular tissue reflects increase the proliferative phase of wound healing. Efem in 1988 reported the increased thickness of granulation tissue in wounds treated with honey (23).

Fibroblast cell is one of most effective and the most important cell in the phenomenon of wound healing that delivers itself on the third day of recovery from the wound. During the proliferation, collagen synthesis amount grows up to 3 weeks continuously. According to the second samples reviews, fibroblast cells amount in the third group was more than the control group and another groups. An inflammatory cell in the control group and group 2 were more than group 3 which show that proliferation phase in group 3 began sooner, while control group and other groups were still in the inflammatory phase (fibroblasts and endothelial cells presence reflects proliferative phase). In the third week, collagen amount in group 3 was more than control and another groups. It seems that honey and nano zinc accelerate collagen synthesis and cause the collagen reached earlier to the maximum level and restructuring phase began faster. Honey provides nutrients and oxygen to fibroblasts through increasing angiogenesis and accelerates the release of oxygen from hemoglobin through acidity generation.

In Massague study conducted in 1990, platelet derived TGF- $\beta 1$ molecules appear in wound place during skin damage for burning and promotes wound healing process (24). TGF- $\beta 1$ increase mitosis power in human dermal fibroblasts (25). TGF- $\beta$ increase angiogenesis process in various tissues through increasing angiogenic expression factors like VEGF (26). TGF- $\beta$ in wound place causes angiogenesis stimulation, the proliferation of fibroblasts, myofibroblasts differentiation, and formation of extracellular matrix. The first goal in wound healing was its tightening quickly. Therefore drugs that modulate the inflammatory response seem appropriate. 
Unsaturated fatty acids are precursors of many lipoic compounds involved in inflammatory reactions. $\omega 3$ and $\omega 6$ fatty acids that are abundant in fish oil and soybean as well as being involved in the formation of lipoic also may be effective in phospholipids membrane. $\omega 9$ fatty acids recover wound rapidly (27). In addition, edema and fibrin clot is thinner. Also, these fatty acids induced the expression of Collagen type III and reduce the expression of cyclooxygenase II rate. Collagen type III is most important Collagen made here. According to abovementioned issues, it can be concluded that topical use of honey and nano zinc on the wound bed has positive effects in accelerate tightening and recovery, layers repair and scar skin and also reduce the scar after healing wounds result from severe burns.

\section{Conclusion}

Histological studies in the present research show protective effect of the combination of nano zinc and honey on repairing and

\section{References}

1. Ronald M, Shelton MAJ, Usaf MC. Aloe vera: its chemical and therapeutic properties. Int J dermatol. 1991; 30(10): 679-3.

2. Subramanian S, Kumar DS, Arolselvan P. Wound healing potential of Aloe vera leaf gel studied in experimental rabbits. Asian J Biochem. 2006; 1(2): 178-85.

3. Jafarzade $\mathrm{H}$, Arabi M, Najafi N, Ahadi A. [Effect of Aloe vera gel on TGF- $\beta$ gene expression in incisional skin wound in $\mathrm{BALB} / \mathrm{c}$ mice]. J Gorgan Uni Med Sci. 2014; 16(3): 1623.(Persian)

4. Boudreau MD, Beland FA. An evaluation of the biological and toxicological properties of Aloe Barbadensis (miller). J Environ Sci Health C Environ Carcinog Ecotoxicol Rev. 2006; 24(1): 103-54. tissue rearrangement on skin ulcers which this reinforcement of rearrangement is with augmenting of the strings of collagen, augmenting of granulation tissue and also the strength of fibroblast cells proliferation on wound area. Application results this research can be base for next researches on volunteers and drug development. Therefore it is suggested that next researches in this field continue.

\section{Acknowledgments}

We wish to thank Islamic Azad University officials of Qom and Borujerd branches and Pathobiology Laboratory, Dr. Aliyari, for his valuable guides during various stages of this work. The research project of the paper approved by Hamadan University of Medical Sciences under ethics committee on Publication Ethics (Code: IR.UMAHA.REC.1395.94).

\section{Conflicts of interest}

The authors declare that there are no conflicts of interest.

5. Heck E, Head M, Nowak D, Helm P, Baxter C. Aloe vera (gel) cream as a topical treatment for outpatient burns. Burns. 1981; 7(4): 291-4.

6. malekhosseini A, Ghaffarzadegan R, Alizadeh SA, Ghaffarzadegan RR, Haji Agaei R, Ahmadlou M. Effect of Aloe vera gel, compared to $1 \%$ silver sulfadiazine cream on second-degree burn wound healing. Complement Med J. 2013; 1(3): 418-28.

7. Ansari M, Alizadeh M, Paknejad M, Khani M, Naeimi S. [Effects of teucrium polium honey on burn wound healing process]. J Babol Univ Med Sci. 2009; 11(3):7-12. (Persian)

8. Riahi S, Imani H, Turkman G, Mofid M, Qoshoni H, Khoshbaten A. [Effect of topical application of Honey in the full-thickness skin wound healing in 
male rats]. Kowsar Med J. 2008; 13(3): 169-78. (Persian)

9. Molan PC. The antibacterial activity of honey: 1. The nature of the antibacterial activity. Bee World. 1992; 73(1): 5-28.

10. Henriques A, Jackson S, Cooper R, Burton N. Free radical production and quenching in Honeys with wound healing potential. J Antimicrob Chemother. 2006; 58(4): 773-7.

11. Subrahmanyam M, Sahapure AG, Nagane NS, Bhagwat VR, Ganu JV. Effect of topical application of Honey on burn wound healing. Ann Burns Fire Disasters. 2001; 14(3): 143-5.

12. Ratner M, Ratner D. Nanotechnology: A Gentle Introduction to the Next Big Idea. 2th ed. New Jersey: Prentice Hall PTR. 2003.

13. Moghadas H, Lafzi A. [Clinical comparison between bone blend with and without doxcycline in the treatment of alveolar vertical defects]. J Dent Sch Shahid Beheshti Uni Med Sci. 1999; 4(16):343-51. (Persian)

14. Barati B, Abbasi H, Omrani MR. The Effects of hydrogen peroxide solution and tetracycline ointment in healing of traumatic facial wounds: A comparative study. Qom Med J. 2007; 1(2): 53-9.

15. Hasanzadeh GHR, Nori A, Hajiabadei M, Soltan K. Javadei A. [The effect of egg yolk on burn wound healing in Rats]. J Gorgan Univ Med Sci. 2005; 7(2):6-10. (Persian)

16. Raghow $\mathrm{R}$. The role of extracellular matrix in postinflammatory wound healing and fibrosis. FASEB J. 1994; 8(11): 823-31.

17. Huang JS,Wang YH, Ling TY, Chuang SS, Frank J, Huang ss. Synthetic TGF$\beta$ antagonist accelerates wound healing and reduces scarring. FASEB J. 2002; 16(10): 1269-70.

18. Hoseinzadeh E, Samargandi MR, Alikhani MY. [Susceptibility constant and death kinetic Escherichia coli and Staphylococcus aurous to Zinc oxide and copper oxide nanoparticles]. J Isfahan Med Sch. 2013; 30(200) 115363. (Persian)

19. Atmaca SE, Gul K, Cicek R. The effect of Zinc on microbial growth. Tr J Med Sci. 1998; 28: 595-7.

20. Hsiao M, Chen S, Shieh D, Yeh C. One-pot synthesis of hollow Au3Cu1 spherical-like and biomineral botallackite $\mathrm{Cu} 2(\mathrm{OH}) 3 \mathrm{Cl}$ flowerlike architectures exhibiting antimicrobial activity. J Phys Chem B. 2006; 110(1): 205-10.

21. Asharani PV, Kahmun GL, Hande MP, Valiyaveettil S. Cytotoxicity and genotoxicity of silver nanoparticles in human cells. ACS Nano. 2009; 24(3): 279-90.

22. Mandal MD, Mandal S. Honey: its medicinal property and antibacterial activity. Asian Pac J Trop Biomed. 2011; 1(2): 154-60.

23. Efem SE. Clinical observation on the wound healing properties of Honey. $\mathrm{Br}$ J Surg. 1988; 75(7): 679-81.

24. Massague J. The transforming growth factor-beta family. Annu Rev Cell Biol. 1990; 6: 597-641.

25. Pertovaara L, Kaipainen A, Mustonen T, Orpana A, Ferrara N, Saksela O, et al. Vascular endothelial growth factor is induced in response to transforming growth factor-beta in fibroblastic and epithelial cells. J Biol Chem. 1994; 4; 269(9): 6271-4.

26. Naghsh N, Aboutalebi F, Karam Seychani S. [Designing a New NanoPlant Composite of Cucurbita pepo for Wound Repair of Skin in Male Albino Mice: A New Nano Approach for Skin Repair]. J Fasa Univ Med Sci. 2013; 3(1): 27-33. (Persian)

27. Malekmohammadi M, Abdol Tehrani $\mathrm{H}$, Aghdami N. Skin structure and wound healing phases. J Dermatol Cosmetic. 2011; 2(4): 229-44. 